\title{
ESTRATEGIAS DE AFRONTAMIENTO QUE PREDICEN MAYOR RESILIENCIA
}

\author{
M. Consuelo Morán-Astorga \\ mcmora@unileon.es \\ María José Finez-Silva \\ Esther Menezes dos Anjos \\ María Cruz Pérez-Lancho \\ José David Urchaga-Litago \\ Gema Vallejo-Pérez
}

Fecha de Recepción: 13 Enero 2019

Fecha de Admisión: 30 Abril 2019

\section{RESUMEN}

Resiliencia y afrontamiento son dos tópicos de gran relevancia en la investigación psicológica. La resiliencia es la capacidad de prosperar y desarrollar nuestro potencial una vez superadas las situaciones traumáticas del devenir personal. Las estrategias de afrontamiento que más comúnmente utilizamos para resolver los problemas que causan estrés son 14. El objetivo de este trabajo fue averiguar qué estrategias de afrontamiento predicen mayor resiliencia. Completaron los cuestionarios de resiliencia y de afrontamiento 734 adultos trabajadores de la provincia de León (51\% mujeres). Se trataron los datos con el SPSS. Se realizaron análisis de regresión lineal, con resiliencia como variable dependiente y las 14 estrategias de afrontamiento como variables predictoras. Los resultados informan que el afrontamiento es un predictor robusto de la resiliencia. Algunas estrategias predicen una mayor resiliencia; otras estrategias nos hacen más vulnerables. Se puede concluir que es de gran importancia que las autoridades educativas (padres, políticos, profesores, etc.) tengan en cuenta la ventaja de incrementar los esfuerzos en la enseñanza-aprendizaje de aquellas estrategias de afrontamiento que nos hacen más fuertes para manejar las situaciones difíciles a las que estamos expuestos en la vida.

Palabras clave: resiliencia; afrontamiento; psicología positiva; aprendizaje

\section{ABSTRACT \\ Coping strategies that predict greater resilience. Resilience and coping are two topics of great relevance in psychological research. Resilience is the ability to grow and develop our potential once the traumatic situations of personal evolution have been overcome. The coping strategies we use to solve the problems that origins stress are 14, the most commonly used. The objective of this work was to find out the coping strategies that predict greater resilience. 734 working adults from the}


province of León completed the resilience and coping questionnaires (56\% women). The data was treated with the SPSS. Linear regression analyzes were performed, with resilience as a dependent variable and the 14 coping strategies as predictor variables. The results report that coping is a robust predictor of resilience. Some strategies predict greater resilience; other strategies make us more vulnerable. it can be concluded that it is of great importance that the educational authorities (parents, politicians, teachers, etc.) take into account the advantage of increasing efforts in the teaching and learning of coping strategies that make us stronger to handle difficult situations to which we are exposed in life.

Keywords: resilience; coping; positive psychology; learning

\section{INTRODUCCIÓN}

La vida en sí misma presenta una gran variedad de retos y desafíos a todos los organismos. Vivir significa ir salvado dificultades. Las personas no somos diferentes en este sentido. Algunas de estas dificultades son livianas, otras son de mayores dimensiones. Bonanno y Mancini (2008) indican que la mayoría de las personas experimentan al menos un evento potencialmente traumático en su vida. La manera de reaccionar a estos eventos diferencia a unas personas de otras (Fletcher y Sarkar, 2013) y más aún cuando el trauma puede ser el resultado. La psicología estudia las diferencias humanas en resiliencia para entender por qué algunas personas son capaces de soportar, incluso prosperar bajo la presión que experimentan en sus vidas, en tanto que otras fracasan y salen perjudicadas o traumatizadas.

Actualmente existe un gran interés en conocer los factores que pueden ser determinantes de la resiliencia. Se ha propuesto que algunas características de personalidad como la seguridad en uno mismo y en la propia capacidad de afrontamiento, tener un propósito significativo en la vida, creer que uno puede influir en lo que le sucede y creer que se puede aprender tanto de las experiencias positivas como de las negativas, favorecerían respuestas resilientes (Morán, Fínez y FernándezAbascal, 2017). También parece que influye el tener un buen autoconcepto y una sana autoestima, o lo que es lo mismo, tener un sesgo positivo en la percepción de uno mismo parece influir y promover un mejor ajuste ante la adversidad (Fínez y Morán, 2014).

En la vida humana, podemos considerar la resiliencia como el conjunto de cualidades personales que nos hacen crecer y desarrollarnos más vigorosamente en la adversidad y tener un funcionamiento óptimo una vez superado el trauma. Se ha demostrado que existen fortalezas humanas que actúan como amortiguadores contra los trastornos mentales y el trauma. Parece existir suficiente evidencia empírica de que determinadas características positivas y fortalezas humanas, como el optimismo, la esperanza, la perseverancia o el valor, entre otras, actúan como barreras contra los trastornos mentales (Morán, Vicente, Sánchez y Montes, 2014). Este modelo que defiende la psicología positiva de personalidad resiliente refuerza la idea de que las personas auténticas controlan mejor su vida (Dos Anjos y Morán-Astorga, 2016).

La resiliencia es vista, más que como la simple recuperación de un trauma, como un desarrollo positivo vigoroso o adaptación a los siguientes periodos de perturbación homeostática. Aunque se pensó que caracteriza a individuos extraordinarios, investigaciones más recientes sobre la adaptación positiva en respuesta a la adversidad extrema, proponen que la resiliencia es relativamente más común entre niños y adolescentes que han estado expuestos a la adversidad, al trauma y al infortunio (Morán-Astorga, 2013). En adultos, la resiliencia podría definirse como la habilidad para mantenerse relativamente sano y con niveles estables de funcionamiento físico y psicológico después de experiencias traumáticas.

Luthar y Cicchetti (2000) definen la resiliencia como un proceso en el que el sujeto exhibe una adaptación positiva a pesar de experiencias que pueden ser adversas o traumáticas. En esta misma 
línea, Manciaux, Vanistendael, Lecomte y Cyrulnik (2001) la definen como la capacidad de las personas para seguir proyectándose en el futuro a pesar de acontecimientos desestabilizadores, de condiciones de vida difíciles y de traumas a veces graves.

La resiliencia, como capacidad para mantener un funcionamiento adaptativo de las funciones físicas y psicológicas en situaciones críticas, no es una condición absoluta ni se adquiere de una vez para siempre. Es el resultado de un proceso dinámico y evolutivo que varía según las circunstancias, la naturaleza del trauma, el contexto y la etapa de la vida. Como todo proceso es un devenir, no significa tanto que una persona es resiliente como que lo es su evolución y el proceso de vertebración de su propia historia vital (Cyrulnik, 2001).

Rizzato y Morán (2013) encuentran, en una muestra de universitarios brasileños, que los que presentan mayores puntuaciones en autoeficacia, más estables emocionalmente y más responsables son más resistentes al estrés y al cansancio emocional.

En un estudio realizado con adolescentes españoles, Fínez y Morán-Astorga (2017) hallaron que aquellos que son capaces resistir, sobreponerse a la adversidad y fortalecerse con la misma, es decir, resilientes, son los que hacen unas evaluaciones más positivas de sí mismos, de su funcionamiento y de su propio entorno.

Existe un acuerdo entre los investigadores en considerar que la manera de afrontar los problemas que en la vida nos causan estrés puede ser uno de los determinantes de la resiliencia (Becoña, 2006). De hecho, Connor y Davidson (2003) definen la resiliencia como una medida de la capacidad que tienen los organismos vivos para enfrentarse con el estrés.

Respecto al afrontamiento, la teoría de Lazarus y Folkman (1984) es la que ha dominado en este campo durante las últimas décadas. En esta teoría, se define el afrontamiento como los intentos cognitivos, emocionales y conductuales para manejar demandas que son evaluadas como exigentes 0 que exceden la propia capacidad del individuo para adaptarse. El afrontamiento es cualquier esfuerzo para manejar el estrés y se evidencia su ejecución mediante las acciones de las personas para evitar ser dañadas por las adversidades de la vida. Estas acciones han sido conceptualizadas como estrategias de afrontamiento (Morán, Méndez, González-Ramírez y Landero-Hernández, 2014).

Otra característica del afrontamiento es que es una respuesta susceptible de ser aprendida frente a las situaciones problemáticas. También que el afrontamiento requiere un esfuerzo, unas veces consciente, otras no, pero requiere un esfuerzo dirigido a manejar la situación que, en ocasiones, cuando no se puede cambiar, requiere un esfuerzo para adaptarse a ella (Morán, 2009).

Se denominan estrategias de afrontamiento a las acciones de los individuos tendentes a frenar, amortiguar y, si es posible, anular los efectos de las situaciones amenazantes. Tomando como referencia teórica los trabajos de Lazarus, otros autores (Carver, Scheier y Weintraub, 1989) han desarrollado un modelo multidimensional del afrontamiento. Estos últimos autores crearon un cuestionario denominado COPE para evaluar el afrontamiento y encontraron una serie de estrategias, conceptualmente diferentes, que la mayoría de las personas utilizamos para hacer frente a los problemas que nos causan estrés. Posteriormente Carver (1997) realiza una versión breve de ese cuestionario con solo 28 ítems: el Brief COPE. Este cuestionario, con dos ítems por escala, evalúa 14 estrategias de afrontamiento.

El Brief COPE (COPE-28 en su versión española) consta de 14 subescalas que evalúan las 14 estrategias de afrontamiento (para una mayor descripción ver Morán, Landero y González, 2010): Afrontamiento activo, Planificación, Apoyo instrumental, Uso de apoyo emocional, Auto-distracción, Desahogo, Desconexión, Reinterpretación positiva, Negación, Aceptación, Religión, Uso de sustancias: (alcohol, medicamentos), Humor y Auto-inculpación. 
En este trabajo nos planteamos como objetivo averiguar qué estrategias de afrontamiento predicen una mayor resiliencia para superar con éxito las situaciones difíciles de la vida.

\section{MÉTODO}

\section{Participantes}

Fueron 734 adultos de la provincia de León, trabajadores de empresa privada (el 74\%) y de empresa pública el resto. El $51 \%$ eran mujeres. La distribución por puesto de trabajo fue: el $34 \%$ ocupaban puestos de dirección, jefatura, profesores, médicos o enfermeras (en general puestos para los que se requiere poseer un título universitario. El 31\% trabajaban en puestos para los que se les pidió el título de bachiller o de formación profesional. El resto $35 \%$ realizaban trabajos de limpieza o de peón u otros puestos que no se requirió titulación. Por nivel de formación, la distribución fue: El $43 \%$ tenían estudios universitarios, el 38\% eran bachilleres o habían cursado formación profesional y un 19\% tenían estudios de escolaridad básica.

\section{Instrumentos de medida}

Se aplicó la Connor-Davidson Escala de Resiliencia-25 (CD-RISC) de Connor \& Davidsosn (2015). La CD-RISC es una escala de 25 ítems donde se refleja el grado de acuerdo a cómo se ha sentido durante el último mes calificándolo en una escala de 5 puntos (de $0=$ nunca a $4=c a s i$ siempre, con puntuaciones intermedias). La mayor puntuación total obtenida indica una mayor resiliencia. Son ejemplo de ítems: 1) Soy capaz de adaptarme cuando ocurren cambios; 11) Creo que puedo lograr mis objetivos, incluso si hay obstáculos; y 22) Creo que controlo mi vida. La consistencia interna para la escala en el estudio de 2003 de los autores dio un Alfa de Cronbach $=, 89$ en adultos normales de USA; en nuestro estudio, el Alfa de Cronbach fue de ,86.

Y el cuestionario COPE-28, versión española del Brief COPE de Carver (1997) realizada por Morán y Manga (documento sin publicar). Es un inventario de 28 ítems y 14 subescalas que se responde en una escala ordinal tipo Likert con 4 alternativas de respuesta (de 0 a 3 ), entre " 0 = nunca hago esto" a "3 = hago siempre esto" con puntuaciones intermedias.

\section{RESULTADOS}

Para conocer en qué medida las estrategias de afrontamiento predicen resiliencia, realizamos un análisis de regresión con las estrategias de afrontamiento como variables predictoras y con la puntuación en la escala de resiliencia como variable dependiente.

Tabla 1. Resumen del modelo

\begin{tabular}{|c|c|c|c|c|c|c|c|c|}
\hline \multirow[b]{2}{*}{ Modelo } & \multirow[b]{2}{*}{$\mathrm{R}$} & \multirow[b]{2}{*}{$\begin{array}{c}\mathrm{R} \\
\text { cuadrado }\end{array}$} & \multirow[b]{2}{*}{$\begin{array}{c}\mathrm{R} \\
\text { cuadrado } \\
\text { ajustado }\end{array}$} & \multicolumn{5}{|c|}{ Estadísticos de cambio } \\
\hline & & & & $\begin{array}{c}\text { Cambio } \\
\text { en R } \\
\text { cuadrado }\end{array}$ & $\begin{array}{c}\text { Cambio } \\
\text { en F }\end{array}$ & gll & $\mathrm{gl} 2$ & $\begin{array}{c}\text { Sig. } \\
\text { Cambio } \\
\text { en F }\end{array}$ \\
\hline 1 &, $585 \mathrm{a}$ &, 342 & 329 & ,342 & 26,630 & 14 & 718 &, 000 \\
\hline
\end{tabular}

a. Predictores: (Constante), Uso de sustancias, Autodistracción, Religión, Planificación, Apoyo Social, Humor, Negación, Autoinculpación, Aceptación, Desahogo, Reevaluación Positiva, Desconexión, Afrontamiento Activo, Apoyo Emocional.

b. Variable dependiente: Resiliencia. 
Las estrategias de afrontamiento sí están asociadas o predicen la resiliencia, y esta asociación es fuertemente significativa $(F=26,63$; sig. $=, 000)$ como puede verse en la tabla 1 .

En la tabla 2 vemos qué estrategias de afrontamiento predicen, están asociadas de manera significativa con resiliencia y el sentido de esta asociación. Las estrategias de afrontamiento asociadas a resiliencia son aquellas cuya significación (Sig.) es menor 0 igual a ,05. La asociación será más fuerte cuando t es más alta.

Es afrontamiento activo es la estrategia que más influye en la resiliencia $(t=8,615$; sig. =,000), y esta influencia es positiva.

También influyen positivamente, con influencia fuerte, Reevaluación positiva y aceptación del problema ( $t=5,334$; sig. =,000 y $t=4,194$; sig. =,000 respectivamente).

Asimismo, influyen positivamente en resiliencia las estrategias de humor y religión cuyas puntuaciones pueden verse en la misma tabla.

Tabla 2. Análisis de regresión con Resiliencia como la variable dependiente y las 14 estrategias de afrontamiento como variables predictoras.

\begin{tabular}{l|c|c|c|c|c} 
& \multicolumn{2}{c}{$\begin{array}{c}\text { Coeficientes no } \\
\text { estandarizados }\end{array}$} & \multicolumn{2}{c}{$\begin{array}{c}\text { Coeficientes } \\
\text { estandarizados }\end{array}$} & \\
Modelo & B & $\begin{array}{c}\text { Error } \\
\text { estándar }\end{array}$ & Beta & t & Sig. \\
\hline $1 \quad$ (Constante) & 43,920 & 2,251 & & 19,508 &, 000 \\
\hline Afrontamiento Activo & 3,298 &, 383 &, 316 & 8,615 &, 000 \\
\hline Planificación &, 414 &, 331 &, 046 & 1,253 &, 211 \\
\hline Apoyo Emocional &,- 114 &, 308 &,- 014 &,- 371 &, 711 \\
\hline Apoyo Social &,- 142 &, 347 &,- 016 &,- 410 &, 682 \\
\hline Religión &, 473 &, 236 &, 063 & 2,004 &, 045 \\
\hline Reevaluación Positiva & 1,634 &, 306 &, 189 & 5,334 &, 000 \\
\hline Aceptación & 1,424 &, 339 &, 142 & 4,194 &, 000 \\
\hline Negación &, 366 &, 304 &, 041 & 1,205 &, 228 \\
\hline Humor &, 792 &, 227 &, 120 & 3,491 &, 001 \\
\hline Autodistracción &,- 031 &, 249 &,- 004 &,- 126 &, 900 \\
\hline Autoinculpación &,- 353 &, 286 &,- 042 & $-1,233$ &, 218 \\
\hline Desconexión & $-1,042$ &, 364 &,- 103 & $-2,868$ &, 004 \\
\hline Desahogo &,- 807 &, 276 &,- 099 & $-2,926$ &, 004 \\
\hline Uso de sustancias &,- 293 &, 346 &,- 028 &,- 846 &, 398 \\
\hline
\end{tabular}

a. Variable dependiente: Resiliencia

En la tabla 2 también vemos las estrategias de afrontamiento que están asociadas negativamente con resiliencia: son desconexión y desahogo con puntuaciones $\mathrm{t}=-2,868$; sig. $=, 004$ y t $=-2,926$ y sig. $=, 004)$.

\section{CONCLUSIONES Y DISCUSIÓN}

Las estrategias de afrontamiento predicen el $34 \%$ de la variabilidad de resiliencia, y esta predicción es robusta.

Algunas estrategias la predicen en positivo, esto es, cuanto más se incrementa su uso, más resi- 
liente llega a ser la persona; otras lo hacen en negativo y significa que utilizarlas convierte a esas personas en más vulnerables, menos resilientes.

Las siguientes estrategias predicen positivamente ser más resilientes:

Afrontamiento activo es la estrategia que más influye en la resiliencia. Utilizar afrontamiento activo significa que las personas que realizan acciones dirigidas a eliminar al estresor, incrementando los esfuerzos para reducirlo o acabar con él, les hace ser más resilientes a la adversidad, superando mejor los traumas y proyectando un futuro más fértil.

Reinterpretación positiva es otra importante estrategia que acrecienta la resiliencia, lo que significa que buscar el lado positivo y favorable del problema e intentar mejorar y crecer a partir de la situación ayuda en buena manera a manejar las situaciones difíciles y traumáticas y a salir fortalecido de ellas.

Aceptación significa reconocer y aceptar el hecho de lo que está ocurriendo, de que tenemos un problema, de que es real, esto es siempre el primer paso para intentar solucionarlo.

Humor significa hacer bromas sobre el estresor o reírse de las situaciones estresantes, haciendo burlas de la misma, esta estrategia también incrementa la resiliencia.

Religión: La tendencia a volver hacia la religión en momentos de estrés, tener la creencia de que dios o la suerte pueden ayudar en momentos críticos también ayuda a soportar las situaciones traumáticas superándolas y adaptarse mejor en el futuro.

Aunque hay dos estrategias que disminuyen la resiliencia, estas son:

Desahogo: Contar nuestra experiencia traumática, tender a expresar o descargar esos sentimientos nos hace aumentar la conciencia del propio malestar emocional, y esto no ayuda.

Desconexión conductual: significa que cuando reducimos los esfuerzos para tratar con el estresor, incluso renunciando al esfuerzo para lograr las metas con las cuales se interfiere al estresor nos hace ser menos resilientes.

Otros investigadores (Cambil-Contreras, 2015) también hallaron una correlación positiva entre la resiliencia y las estrategias de afrontamiento centradas en el problema (Afrontamiento activo, reinterpretación positiva, aceptación entre ellas) y negativa entre resiliencia y estrategias de afrontamiento centradas en la emoción (desahogo y desconexión conductual entre otras).

Benavente-Cuesta y Quevedo-Aguado (2018) destacan la relación entre las dimensiones de la resiliencia y las del bienestar psicológico, así como la relación de éstas con dos de las estrategias de afrontamiento: Focalización en la solución de problemas y reevaluación positiva. Respecto a la primera afirmación, se podría interpretar que al aumentar los niveles de las distintas subdimensiones de la resiliencia, aumentan las dimensiones del bienestar psicológico, sin poder determinar ningún tipo de relación causal. Por otro lado, estos resultados confirman que las personas resilientes, así como aquellas que gozan de un elevado estado de bienestar, emplean estrategias de afrontamiento específicas, en este caso, la focalización en la solución de problemas y la reevaluación positiva.

\section{REFERENCIAS BIBLIOGRÁFICAS}

Becoña, E. (2006). Resiliencia: Definición, características y utilidad del concepto. Revista de Psicopatología Clínica, 11(3), 125-146.

Benavente-Cuesta, M.H. y Quevedo-Aguado M.P. (2018). Resiliencia, bienestar psicológico y afrontamiento en universitarios atendiendo a variables de personalidad y enfermedad. Revista de Psicología y Educación / Journal of Psychology and Education, 13(2), 99-112

Cambil-Contreras, M. (2015). Resiliencia, estrategias de afrontamiento y enfoques de aprendizaje, como aspectos de la competencia aprender a aprender con estrés en el contexto de enseñanzaaprendizaje universitario (Tesis doctoral). Universidad de Granada (España). 
Carver, C. S. (1997). You want to measure coping but your protocol's too long: Consider the Brief COPE. International Journal of Behavioral Medicine, 4(1), 92-100.

Carver, C. S., Scheier, M. F. \& Weintraub, J. K. (1989). Assessing coping strategies: A theoretically based approach. Journal of Personality and Social Psychology, 56, 267-283.

Connor, K. M., \& Davidson, J. R. T. (2003). Development of a new resilience scale: The ConnorDavidson Resilience Scale (CD-RISC). Depression and Anxiety, 18, 71-82.

Connor, K. M., \& Davidson, J. R. T. (2015). Escala de Resiliencia de Connor-Davidson (CD-RISC). Disponible https://www.researchgate.net/publication/277139516_Escala_de_Resiliencia_de_ConnorDavidson_CD-RISC [accessed Apr 04 2019].

Cyrulnik, B. (2001). Los Patitos feos: la resiliencia: una infancia infeliz no determina la vida. Madrid: Gedisa.

Dos Anjos, E. M. y Morán-Astorga, M.C. (2016). A personalidade resiliente: Uma conceptualização teórica. International Journal of Developmental and Educational Psychology INFAD Revista de Psicología, 1 (2), 151-156.

Fínez, M. J. y Morán-Astorga, M.C. (2017). Resiliencia y autovaloraciones esenciales: estudio comparativo en adolescentes y jóvenes. Psychology, Society, \& Education, 9(3), 347-356. https://doi.org/0.25115/psye.v9i3.857

Fínez, M.J. y Morán-Astorga, M.C. (2014). Resiliencia y autoconcepto: su relación con el cansancio emocional en adolescentes. International Journal of Developmental and Educational Psychology. INFAD Revista de Psicología, 1(6), 289-296. https://doi.org/10.17060/ijodaep.2014.n1.v6.746.

Fínez, M.J. y Morán-Astorga, M.C. (2015). La resiliencia y su relación con salud y ansiedad en estudiantes españoles. International Journal of Developmental and Educational Psychology INFAD Revista de Psicología, 1 (1), 409-416. http://dx.doi.org/10.17060/ijodaep.2015.n1.v1.90.

Fletcher, D. y Sarkar, M. (2013). Psychological resilience: A review and critique of definitions, concepts, and theory. European Psychologist, 18(1), 12-23. D0I: 10.1027/1016-9040/a000124

Lazarus, R. y Folkman, S. (1984). Estrés y procesos cognitivos. Barcelona: Martínez Roca.

Luthar, S.S. y Cicchetti, D. (2000). Child Dev., 71(3), 543-562.

Manciaux, M., Vanistendael, S., Lecomte, J. y Cyrulnik, B. (2003). La resiliencia: estado de la cuestión. En M. Manciaux (comp.), La resiliencia: resistir y rehacerse. Madrid: Gedisa.

Manga, D., Ramos, F. y Morán-Astorga, M.C. (2004). The Spanish norms of the NEO-Five Factor Inventory: New data and analyses for its improvement. International Journal of Psychology and Psychological Therapy, 4 (3), 639-648.

Morán-Astorga, M.C. (2009). Estrés, burnout y mobbing. Recursos y estrategias de afrontamiento. Salamanca: Amarú.

Morán-Astorga, M.C. (2005). Personalidad, afrontamiento y burnout en profesionales de atención a personas con discapacidad intelectual, Siglo Cero, 213 (36), 30-39.

Morán-Astorga, M.C. (2013). Una psicología en el ámbito laboral. Competencias para recursos humanos. Salamanca: Amarú.

Morán-Astorga, M.C., Fínez, M. J. y Fernández-Abascal. E. (2017). Sobre la felicidad y su relación con tipos y rasgos de personalidad. Clínica y Salud, 28, 59-63. http://dex.doi.org/:10.1016/i.clysa.2016.11.003

Morán-Astorga, M.C., Landero, R. y González, M. (2010). COPE-28: un análisis psicométrico de la versión en español del Brief COPE. Universitas Psychologica, 9 (2), 23-40.

Morán-Astorga, M.C. y Schulz, R. (2008). Influencia del optimismo y del afrontamiento en el bienestar laboral. Capital Humano, 225, 86-92. 
Morán-Astorga, M.C., Méndez, L. M., González-Ramírez, M. T. y Landero-Hernández, R. (2014). Evaluación de las propiedades psicométricas del Brief COPE, su relación con el NEO PI-R y diferencias de género en Brasil. Universitas Psychologica 13(4), 15-30. DOI: 10.11144/Javeriana.UPSY13-4.eppb

Morán-Astorga, M.C. Vicente, F., Sánchez-Cabaco, A. y Montes, E. (2014). Psicología positiva. Antigua 0 nueva concepción. International Journal of Developmental and Educational Psychology, 3, (1), 439-450. http://dx.doi.org/10.17060/ijodaep.2014.n1.v3.522.

Rizzato, S. C. y Morán, M. C. (2013). Emprendedorismo e personalidade: o perfil em estudantes brasileiros. Revista Psicología Organizações e Trabalho, 13(3), 279-292. 